\section{Fenster in die Nachhaltigkeit}

\author{
Den Erfolg neuer Technologien bedingen eine ganze Reihe von Faktoren. Bei der \\ politischen Unterstützung neuer, umweltfreundlicher Technologien ist ein zen- \\ traler Faktor bislang außer Acht gelassen worden: die Innovationsdynamik am \\ Markt. Wird sie systematisch unter zeitstrategischen Gesichtspunkten analy- \\ siert, so ergeben sich verschiedene Zeiffenster, die je nach Innovationsdynamik \\ politisch unterschiedlich genutzt werden sollten. Werden sie beachtet, wird die \\ Einführung umweltfreundlicher Technologien wesentlich kostengünstiger und \\ konfliktfreier sein als bisher.
}

$I^{-1}$ Von Christian Sartorius und Stefan Zundel nnovationen erfreuen sich in der Umweltpolitik großer Aufmerksamkeit. Sie sind in vielen Fällen eine wirkungsvolle Möglichkeit, die negativen ökologischen Nebenwirkungen etablierter Technologien zu verringern oder sogar zu beseitigen. Sie erlauben die Fortführung unseres von Wachstum geprägten Lebensstils, ohne dass die Umwelt dabei zwangsläufig Schaden nimmt soweit der Anspruch.

In Wirklichkeit ist diese Wirkung von Innovationen jedoch in mehrfacher Hinsicht eingeschränkt. Einerseits erfahren umwelffreundliche Technologien häufig gerade aufgrund der Tatsache, dass sie schädliche Nebenwirkungen nicht externalisieren, einen Wettbewerbsnachteil, der ihre Ausbreitung systematisch behindert. Marktkonforme Regulierungsinstrumente wie Ökosteuern oder Emissionszertifikate wären zur Kompensation dieses permanenten Nachteils grundsätz-

Tabelle 1: Zeitstrategien ökologischer Innovationspolitik

\begin{tabular}{|c|c|c|c|c|}
\hline Ausgangssituation: & Status des & Technologischer & Innovations- & Zeitstrategischer \\
\hline Qualität der Lösungen & technologischen Regimes & Wettbewerb & politisches Ziel & Ansatz \\
\hline \multicolumn{5}{|l|}{ für ein Umweltproblem } \\
\hline vielversprechende & noch stabil & n.a. & Entwicklung von und & Fenstervorbereitung \\
\hline Invention & & & Vielfalt an Alternativen & \\
\hline vielversprechende & noch stabil & n.a. & Entspannung politischen & Fensteröffnung \\
\hline Invention + & & & Drucks durch Forcierung & \\
\hline politischer Druck & & & einer Lösung & \\
\hline wettbewerbsfähige & instabil & alt-neu & Technologischer Übergang & Fensternutzung \\
\hline Innovation & & & ohne Diskriminierung & \\
\hline + vielversprechende & & & von Alternativen & \\
\hline \multicolumn{5}{|l|}{ Alternativen } \\
\hline mehrere & instabil & neu-neu & Vermeidung vorzeitiger & Fensterbeibehaltung \\
\hline wettbewerbsfähige & & & Selektion & \\
\hline Lösungsalternativen & & & & \\
\hline
\end{tabular}

Quelle: eigene Darstellung oder das Fehlen spezifischer Nachfrage als mögliche technisch-ökonomische Gründe für einen Lock-out identifiziert werden. Da technologischer Wandel immer in einem politischen und sozialen Kontext stattfindet, ist es unumgänglich, auch politische Faktoren wie zum Beispiel den institutionellen Kontext, Stärke von Interessengruppen oder Mehrheitsverhältnisse sowie sozial-kulturelle Faktoren wie gesellschaftliche Sorge um ein Umweltproblem oder Akzeptanz einer Lösung mit in die Betrachtung einzubeziehen.

Wenngleich eine vollständige Auflistung relevanter Faktoren nicht möglich ist, so ist ihnen gemeinsam, dass die von ihnen ausgehende, teilweise starke Neigung zum Lock-in starken zeitlichen Schwankungen unterliegt (1). Phasen der Stabilität einer etablierten Technologie wechseln sich mit Phasen der Instabilität $a b$, wobei ein technologischer Wechsel innerhalb instabiler Phasen leichter ist als in stabilen Phasen. Es sind diese Windows of Opportunity oder Zeitfenster, die von der Politik genutzt werden können, um einer umweltfreundlicheren Technologie mit deutlich geringerem Aufwand zum Durchbruch zu verhelfen als es mit einer zeitunspezifischen Strategie möglich wäre.

In diesem Zusammenhang wurden im Rahmen des SUSTIME-Projekts verschiedene, in Tabelle 1 zusammengeführte Zeitstrategien ökologischer Innovationspolitik entwickelt, deren sich die Politik einzeln oder in Kombination bedienen kann, um den erwünschten Effekt zu erzielen (2).

\section{Politische Nutzung von Zeitfenstern}

Der naheliegendste und zunächst am wenigsten aufwändige zeitstrategische Ansatz besteht in der Identifikation und Nutzung bereits bestehender Zeitfenster. Er geht davon aus, dass die technische Lösung eines Umweltproblems grundsätzlich bereits wirtschaftlich darstellbar ist und dass auch in Politik und Gesellschaft der Wille zur Lösung des Problems und eine Offenheit für den Einsatz der vorhandenen Lösungsalternative bestehen.

Ein bekanntes Beispiel hierfür sind die technisch wie ökologisch hocheffizienten GuD-Kraftwerke, die in einigen Bereichen der Stromproduktion bereits wirtschaftlich einsetzbar sind, deren weitergehender Einsatz in Deutschland aber im Moment daran scheitert, dass die Kohle gegenüber dem Erdgas einen umweltpolitisch nicht zu begründenden Vorrang genießt, die Stromproduktion noch immer durch Überkapazitäten gekennzeichnet ist und der Marktzugang für unabhängige 
Stromproduzenten nach wie vor erschwert ist (3). Während sich die Frage der Überkapazitäten aufgrund der Außerbetriebnahme alter Kraftwerke bis spätestens 2010 von alleine lösen wird, ist es eine Frage des politischen Willens, ob durch eine vollständige Liberalisierung der Marktzugang unabhängiger Stromproduzenten weiter erleichtert wird und der Vorrang der Kohle angesichts seiner klimapolitischen Wirkung aufgegeben wird. Das Kioto-Protokoll, sofern es ratifiziert wird, und die Liberalisierungsrichtlinien der EU für Strom und Gas dürften hier als zusätzliche Triebkräfte auf nationaler Ebene eine wichtige Rolle spielen. Die Rolle der Politik bei der Nut- zung von Zeitfenstern ist also die eines Koordinators, der alle relevanten Faktoren hinsichtlich ihrer instabilen Phasen aufeinander abstimmt (vgl. zu weiteren Beispielen für Fensternutzungsstrategien die Beiträge von Sartorius zur mobilen Brennstoffzelle und von Haum und Nill zu energieeffizienten Wohngebäuden in diesem Heft).

Kann ein technisch-ökonomisches Zeitfenster nicht von vorne herein identifiziert werden, so kann die Politik ein aktiveres Vorgehen in Erwägung ziehen. Insbesondere wenn eine vielversprechende, aber noch nicht marktreife technische Lösung für das vorliegende Umweltproblem existiert, kann die Entstehung eines Zeit- fensters dadurch unterstuitzt beziehungsweise vorbereitet werden, dass entsprechende spezifische Forschungs- und Entwicklungsanreize gesetzt werden.

Als Beispiel sind hier die gezielten Forschungsprogramme zur Steigerung des Wirkungsgrades und der kostengünstigeren Produktion von Solarzellen in den 1980er Jahren zu sehen. Auch die Entwicklung von Nischenmärkten wäre dieser Strategie zuzuordnen. Ebenfalls zum Bereich der Fenstervorbereitung zählen solche Bemühungen, die darauf abzielen, das institutionelle Umfeld an die Erfordernisse der neuen Technologie anzupassen.

\section{Zeitstrategien für den Umgang mit Verpackungskunststoffen in Deutschland}

D

Von Daniel Weiner und Stefan Zundel ie Verwertung von Verpackungskunststoffen, in Deutschland durch das Duale System Deutschland (DSD) und die Deutsche Gesellschaft für Kunststoffrecycling (DKR) organisiert, wurde vielfach als ein wettbewerbsfeindliches System zur Förderung des Kunststoffrecyclings kritisiert. Durch fehlende Verwertungskapazitäten in den Anfangsjahren kam es zu "wilden" und unkontrollierten Abfallströmen ins Ausland und der faktische Monopolstatus des DSD wurde wiederholt als Wettbewerbsverzerrung bemängelt. Dabei kann die Kunststoffverwertung im Rahmen der Verpackungsverordnung durchaus auch als ein erfolgreiches Beispiel eines politischen Eingriffs in einen bestehenden Markt gewertet werden.

Die erste umgesetzte Fassung der Verpackungsverordnung schrieb in einer zweistufigen Quotenregelung für 1993 und 1996 vor, dass mindestens neun beziehungsweise 64 Prozent aller auf dem Markt befindlichen Kunststoffverpackungsabfälle stofflich verwertet werden sollen und zu 91 Prozent beziehungsweise 36 Prozent entsorgt werden können. Der Begriff der stofflichen Verwertung umfasste dabei sowohl die werkstoffliche als auch die rohstoffliche Verwertung. Die Industrie kritisierte, dass eine energetische Verwertung von vornherein nicht zulässig und damit ausgeschlossen war.

Dieser Kritik wurde in der ersten Novelle der Verpackungsverordnung von 1998 insoweit entsprochen, als rückwirkend ab 1996 auch die energetische Verwertung zugelassen wurde. Dafür wurde eine zweite (Unter-) Quote eingeführt, die eine rein werkstoffliche Verwertung forderte. Ab 1999 sollten mindestens 60 Prozent aller li- zenzierten Kunststoffverpackungen verwertet werden, wovon 60 Prozent einer werkstofflichen Verwertung zugeführt werden sollten. Der Rest innerhalb der Mindestverwertungsmenge sollte rohstofflich oder energetisch verwertet werden.

Diese Quotierung der Kunststoffverwertung erzwang einen Einstieg in werkstoffliche Verwertungstechnologien, die sich von allein kaum durchgesetzt hätten. Dieser Vorgang kann als Technology forcing charakterisiert werden, das politische System erzwang die Eröffnung eines technologischen Wettbewerbs, in unserem Terminus ein techno-ökonomisches Zeiffenster. Alternativen zur Abfallbeseitigung, die vor Einführung der Verpackungsverordnung lediglich theoretisch existiert haben, mussten innerhalb kurzer Zeit entwickelt und aufgebaut werden, um die geforderten Verwertungsquoten zu erfüllen.

Gleichzeitig funktionierte die Verwertungsquote ökonomisch wie eine Absatzgarantie, die für das werkstoffliche Recycling gewährt wird. Der Verbraucher bezahlte über den grünen Punkt die Errichtung entsprechender Verwertungskapazitäten; er finanziert die Entwicklungskosten und Lernfortschritte solcher Verfahren. Die zugelassenen Verfahren befanden sich in einem geschützten Markt, der in den Anfangsjahren den Alt-Neu Wettbewerb zwischen dem alten Pfad der Deponierung und dem neuen Pfad der Verwertung suspendierte. Gleiches gilt für den Neu-Neu Wettbewerb der Verwertungstechnologien untereinander, indem die lizenzierten Verfahren die Zulieferung von Kunststoffabfällen zugesichert bekamen und die Menge der Kunststoffabfälle die Verwertungskapazitäten überstieg.

Mittlerweile werden etwa 50 Prozent der erfassten
Kunststoffabfälle werkstofflich verwertet und diese Verfahren haben die Kostenführerschaft im Verwertungsbereich. Die wesentlichen Gründe für diese Veränderung sind eine deutliche Verbesserung der Sortierungstechnologien und der technische Fortschritt bei der werkstofflichen Verwertung selbst.

Würde man also heute die Quote, die den Anteil der werkstofflichen Verwertung sichert, streichen, würde sich vermutlich kaum etwas an der Verteilung der Kunststoffabfälle auf die verschiedenen Verwertungswege ändern. So betrachtet hat das DSD durchaus seinen Zweck erfüllt. Es hat eine Innovations- und Diffusionsdynamik bei den werkstofflichen Verfahren in Gang gesetzt, die der politisch gewünschten Priorität dieser Verfahren ökonomisches Leben eingehaucht hat. Mehrere Verwertungsverfahren sind auf der Lernkurve so weit fortgeschritten, dass sie in absehbarer Zeit wettbewerbsfähig werden. Mit der gleichzeitigen Änderung der rechtlichen Rahmenbedingungen, insbesondere die Umsetzung der Technischen Anleitung für Siedlungsabfälle (TASi) 2006, ist ein politischer Schutz des Marktes nur noch begrenzt notwendig. Das techno-ökonomische System ist wieder stabil - die politische Einflussnahme verliert damit in dieser Form ihre Berechtigung, die Fensternutzung ist erfolgreich abgeschlossen. Und da eine

Liberalisierung der Quotierung des Systems dem werkstofflichen Recycling kaum schaden dürfte, wäre mehr Flexibilität im System nicht nur ökonomisch sinnvoll sondern auch ökologisch kaum bedenklich.

\section{Die Autoren}

Dr. Stefan Zundel ist Professor für Volkswirtschaftslehre mit Schwerpunkt Öffentliche Finanzwirtschaft, Energie und Umwelt an der FH Lausitz. Daniel Weiner ist dort wissenschaftlicher Mitarbeiter.

Kontakt: FH Lausitz, FB Wirtschaftswissenschaften, Großenhainer Straße 57, 01968 Senftenberg. Tel. 03573-85733, Fax -09, E-Mail: stzundel@fh-lausitz.de,dweiner@fh-lausitz.de 


\section{- Aktive Fensteröffnung}

Ist bei Vorliegen einer vielversprechenden, aber noch nicht marktreifen technischen Alternative der Druck zur Lösung eines Umweltproblems zu groß, um auf die eher mittelfristig wirksame Strategie der Fenstervorbereitung und -nutzung setzen zu können, dann bietet sich als weitere Strategiealternative die aktive Öffnung des Fensters an. Dabei beschränkt sich die Politik nicht mehr auf die Schaffung der notwendigen Voraussetzungen und Rahmenbedingungen, sondern greift durch entsprechende Ge- oder Verbote direkt in den Technologiewettbewerb ein.

Eine Form der aktiven Fensteröffnung ist unter dem Begriff des Technology forcing bekannt. Sie ist meist durch politischen Druck motiviert und wurde beispielsweise im Rahmen des kalifornischen Zero Emission Vehicle Mandate praktiziert, wo der Gesetzgeber auf der Grundlage eines vielversprechenden, batteriebetriebenen Prototyps einer Firma die gesamte Branche dazu verpflichten wollte, längerfristig zunehmende Anteile der gesamten Fahrzeugflotte in batteriebetriebener Form auszuliefern. Zumindest im Hinblick auf das batteriebetriebene Fahrzeug muss dieser Versuch als gescheitert angesehen werden.

Als aufwändig, aber erfolgreich muss demgegenüber der Versuch Japans angesehen werden, bei der Chlor-Alkali-Elektrolyse einen vollständigen Übergang vom umweltschädlichen Amalgam-Verfahren zum ökologisch und inzwischen auch ökonomisch überlegenen Membranverfahren zu vollziehen. Generell bleibt festzuhalten, dass die Kosten und das Risiko der aktiven Fensteröffnung deutlich höher sind als bei Fenstervorbereitung und -nutzung. So geschieht die aktive Fensteröffnung unter dem Vorbehalt, dass sich der technischwirtschaftliche Fortschritt wie erwartet entwickelt und die ökologischen Nebenwirkungen der neuen Technologie tatsächlich geringer sind. Da dies aufgrund ökonomischer oder ökologischer Fehleinschätzungen nicht immer der Fall ist, ist genaues Controlling unbedingte Voraussetzung für den Erfolg. Beispielsweise hat sich die prognostizierte Verknappung von Deponieraum als einer der ursprünglichen Hauptbeweggründe zur Einführung von Kunststoffrecycling im nachhinein als falsch erwiesen (vgl. den Beitrag von Weiner und Zundel in diesem Heft).

\section{- Technologiewettbewerb}

Die bisherige Darstellung konzentrierte sich auf den Wettbewerb zwischen einer etablierten und einer neuen Technologie als Anlass für eine umweltpolitisch motivierte Intervention und damit die Wahl einer der zuvor beschriebenen Strategien. Darüber hinaus ist jedoch häufig auch der Wettbewerb zwischen mehreren neuen Technologiealternativen relevant.

In diesem Fall stellt sich zusätzlich die Frage, wie sich das Auftreten von Fenstern auf die Selektion zwischen den Alternativen zur alten Technologie auswirkt. Kleine Vorteile für eine der Alternativen, beispielsweise ein zeitlicher Vorsprung oder die bessere Anpassung an das institutionelle Umfeld, können hier selbst dann zum Ausscheiden der anderen Alternative führen, wenn diese sich später als ökologisch und ökonomisch überlegen erwiesen hätte. Die Herausforderung für die Politik besteht bei dieser Strategie darin, dass die Zeitfenster für beide neuen Technologien so koordiniert werden, dass die Startchancen für beide gleich groß sind. Ist diese Herangehensweise aufgrund des zu unterschiedlichen Entwicklungsgrades beider Technologien zu zeit- oder ressourcenaufwändig, kann der Wettbewerb auf ein späteres Fenster verschoben werden.

Grundsätzlich gilt zu bedenken, dass die Öffnung oder Aufrechterhaltung eines Zeitfensters zwar zu einer Erhöhung technologischer Vielfalt und Variabilität führt, dass sie letztlich aber nicht nur die Selektion der am besten geeigneten Technologie unterbindet, sondern neben Skalen-, Lern- und

Kooperative Umwelt-
Governance -
Am Beispiel Integrierte
Produktpolitik
ist das Thema
des Informationsdienstes
Ökologisches Wirtschaften
3-4/04
inklusive Spezial:
Bildung für
nachbaltige Entwicklung
Wenn Sie potenzielle Beiträge haben,
wenden Sie sich bitte
an die Redaktion.

Netzwerkeffekten eine ganze Reihe weiterer Wirkmechanismen außer Kraft setzen, die die Grundlage unseres Wohlstandes bilden.

Im Streben nach nachhaltigem Wohlstand kommt es darauf an, mit Zeitfenstern strategisch Ressourcen effizient in menschliches Wohlergehen zu transformieren, den genutzten Transformationsapparat aber flexibel zu halten, um auf eventuelle Nebenwirkungen schnell reagieren zu können. Dabei weisen nicht nur die dafür verfügbaren Zeitstrategien ein hohes Maß an Dynamik auf; auch die politischen Instrumente müssen flexibel sein und der Dynamik Rechnung tragen.

\section{Anmerkungen}

(1) Vgl. hierzu z.B. Zundel, S./ Erdmann, G./ Nill, J., Sartorius, C.,/ Weiner, D.: Zeitstrategien ökologischer Innovationspolitik der Forschungsansatz. In: Horbach, J./ Huber, J./ Schulz, T. (Hrsg.): Nachhaltigkeit und Innovation. Rahmenbedingungen für Umweltinnovationen. München 2003, S. 55-88 sowie die Broschüre Zeitstrategien in der Umwelt- und Innovationspolitik. IÖW/ FH Lausitz/ TU Berlin, Berlin 2004.

(2) Das Projekt Innovation, Zeit, Nachhaltigkeit. Zeitstrategien ökologischer Innovationspolitik (SUSTIME) wurde im Rahmen des Förderschwerpunkts Rahmenbedingungen für Innovationen zum nachhaltigen Wirtschaften (:[riw]) vom Bundesforschungsministerium gefördert und im Verbund von der FH Lausitz (Koordination), der TU Berlin, dem Institut für ökologische Wirtschaftsforschung (IÖW) und MERIT Maastricht durchgeführt.

(3) Zu diesem und den weiteren Beispielen vgl. Zundel, S./ Sartorius, C. (Hrsg.): Time strategies in innovation policy towards sustainability, Cheltenham (im Erscheinen).

\section{Die Autoren}

Dr. Christian Sartorius war bis vor kurzem wissenschaftlicher Mitarbeiter an der TU Berlin. Dr. Stefan Zundel ist Professor für Volkswirtschaftslehre mit Schwerpunkt Öffentliche Finanzwirtschaft, Energie und Umwelt an der FH Lausitz.

Kontakt: FH Lausitz, FB Wirtschaftswissenschaften, Großenhainer Straße 57, 01968 Senftenberg. Tel. 03573-85733, Fax -09, E-Mail: stzundel@fh-lausitz.de, c.sartorius@tu-berlin.de 
(c) 20I0 Authors; licensee IÖW and oekom verlag. This is an article distributed under the terms of the Creative Commons Attribution Non-Commercial No Derivates License (http://creativecommons.org/licenses/by-nc-nd/3.o/), which permits unrestricted use, distribution, and reproduction in any medium, provided the original work is properly cited. 